\title{
Observation of whispering gallery modes in cathode luminescence in $\mathrm{TiO}_{2}: \mathrm{Eu}^{3+}$ microspheres
}

\author{
Makoto Tomita ${ }^{a)}$ and Kouki Totsuka \\ Department of Physics, Faculty of Science, Shizuoak University, 836 Ohya, Suruga-ku, \\ Shizuoka 422-8529, Japan \\ Hiroshi lkari \\ Department Physics, Faculty of Education, Shizuoak University, 836 Ohya, Suruga-ku, \\ Shizuoka 422-8529, Japan
}

Kenji Ohara and Hidenori Mimura

Research Institute of Electronics, Shizuoka University, 3-5-1 Johoku, Hamamatsu 432-8011, Japan

Hideyuki Watanabe

Diamond Research Center, Advanced Industrial Science and Technology, 1-1-1 Umezono, Tsukuba 305-8568, Japan

Hiroshi Kume

National Institute for Environmental Studies, 16-2 Onogawa, Tsukuba 305-8506, Japan and Research Institute of Electronics, Shizuoka University, 3-5-1 Johoku, Hamamatsu 432-8011, Japan

Takahiro Matsumoto

Research and Development Center, Stanley Electric Corporation, 5-9-5 Tokodai, Tsukuba 300-2635, Japan and Research Institute of Electronics, Shizuoka University, 3-5-1 Johoku, Hamamatsu 432-8011, Japan

(Received 23 April 2006; accepted 23 June 2006; published online 11 August 2006)

\begin{abstract}
We report cavity enhanced periodic structures in cathode luminescence spectra in $\mathrm{TiO}_{2}$ : $\mathrm{Eu}^{3+}$ microspheres ranging from 6.2 to $12.2 \mu \mathrm{m}$ in diameter. The spectral structures observed at the accelerating voltage below $10 \mathrm{kV}$ are attributed to the lower order whispering galley modes, while the additional components appearing at $20 \mathrm{kV}$ are to the higher order modes within the microsphere. These observations illustrate that the basic properties of phosphors can be advanced from the point of view of cavity quantum electrodynamics by fabricating a microcavity structure onto a single phosphor crystal. (C) 2006 American Institute of Physics. [DOI: 10.1063/1.2335578]
\end{abstract}

In the study of cathode phosphors, a lot of materials have been synthesized and their basic characteristics have been fully examined. ${ }^{1}$ Rare-earth and transition metals are widely used as practical phosphors in modern light emitting devices, while the rapidly developing technologies demand more sophisticated phosphors. For example, since the accessible voltage and current in field emission displays are restricted by several factors, a cathode phosphor with a low working voltage is desired. Along with the traditional method based on solid state reactions, an alternative approach based on sol-gel process has also been applied to fabricate highly pure nanopowders or homogeneous thin film from organic precursors. ${ }^{2,3}$

Most of the previous investigations on phosphors have been devoted to the material research. On the other hand, the emission property is relevant not only to the material but also to the radiation fields interacting with the emission center. This quantum-electrodynamic (QED) effect is known as the Purcell effect, ${ }^{4}$ that is, the luminescence from rare-earth atoms or molecules may be alternatively enhanced or inhibited, depending on whether or not the emission spectrally coincides with the cavity resonances. ${ }^{5-7}$ Here, we discuss a novel concept of cathode phosphor in order to improve the basic properties, such as emission efficiency, color purity, and lifetime from point of view of the QED effect by fabri-

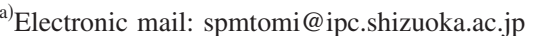

cating a microcavity structure onto a single phosphor crystal. Specifically, we prepared high quality $\mathrm{Eu}^{3+}$ doped $\mathrm{TiO}_{2}$ dielectric spheres and observed cavity enhanced periodic structures in the cathode luminescence (CL) spectra, which are attributed to whispering galley modes ${ }^{5,6,8,9}$ (WGMs) within the spheres. The spherical cavity enhanced effects in photoluminescence, ${ }^{5,10}$ light scattering, and nonlinear optics or the effects associated with other morphological cavity structures $^{11-13}$ have widely been observed in optical experiments. Although these effects have potentialities to develop phosphors, such an effect has not been reported so far in cathode luminescence.

The $\mathrm{Eu}^{3+}$ doped $\mathrm{TiO}_{2}$ microspheres were prepared by a hydrolysis process of alkoxide droplets. ${ }^{14,15}$ First, $\mathrm{Ti}\left(\mathrm{OC}_{4} \mathrm{H}_{9}\right)$ was dissolved at $0.1 \mathrm{~mol} / l$ in a mixed solvent of $n$-octanol and acetonitrile, which contained hydroxy propyl cellulose as the surface stabilizer. Water dissolved in ethanol was added into the mixed solvent, and the subsequent hydrolysis process of alkoxied droplets resulted in hydroxide particles. The sphere size was controlled through the concentration of hydroxy propyl cellulose. In order to dope $\mathrm{Eu}^{3+}$ ion, the microsphere was dipped in aqueous solution of europium nitrate $\left[\mathrm{Eu}\left(\mathrm{NO}_{3}\right)_{3}\right]$. The doped spheres were then crystallized into a stable phase. The cathode luminescence was measured by an experimental setup based on a scanning electron microscope. The $\mathrm{TiO}_{2}: \mathrm{Eu}^{3+}$ microsphere was placed on a metal substrate and set at one of the focal points of the elliptical 


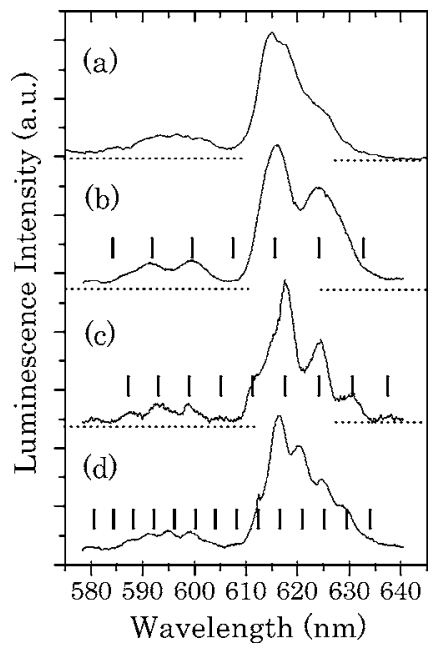

FIG. 1. Cathode luminescence spectra from $\mathrm{TiO}_{2}: \mathrm{Eu}^{3+}$ (a) in the form of agglomerated irregular powders; (b), (c), and (d) are from microspheres of $d=6.2,8.1$, and $12.2 \mu \mathrm{m}$ in diameter, respectively. The vertical lines in (b), (c), and (d) indicate the free spectral rage of $\Delta \nu=6.5,5.0$, and $3.3 \mathrm{THz}$, respectively.

mirror, while the emitted light from the single microsphere was collected from another focal point by using an optical fiber. The luminescence from the fiber was led into a $30 \mathrm{~cm}$ polychrometer and the spectra were measured by a cooled charge-coupled device camera. The spectral resolution was $0.5 \mathrm{~nm}$ and the accelerating voltage was varied from 6 to $20 \mathrm{kV}$.

Figure 1 shows CL spectra from $\mathrm{TiO}_{2}: \mathrm{Eu}^{3+}$ observed at the accelerating voltage of $10 \mathrm{kV}$. Figure 1 (a) is the spectrum from agglomerated irregular shaped powders, where the peaks at 595 and $620 \mathrm{~nm}$ correspond to the transitions of ${ }^{5} D_{0}$ to ${ }^{7} F_{1}$ and ${ }^{5} D_{0}$ to ${ }^{7} F_{2}$ in $\mathrm{Eu}^{3+}$, respectively. Figs. 1(b) $-1(\mathrm{~d})$ are CL spectra from well shaped microsphere. In contrast to the irregular powders, these spectra show periodic structures superimposed over the normal broadband emission. The vertical lines in Figs. 1(b)-1(d) indicate the free spectral rage of $\Delta \nu=6.5,5.0$, and $3.3 \mathrm{THz}$, respectively. When the sphere size is increased from 6.2 to $12.2 \mu \mathrm{m}$, the free spectral range between peaks decreases. We consider that these spectral features arise from cavity enhancement effect in the spontaneous emission by WGM resonance in the microspheres. The WGMs are assigned by radial order number $n$, angular number $l$, azimuth number $m$, and polarization indices, TE or $\mathrm{TM}^{8,9}$ similar to those used in a simple atomic system. For the large mode numbers, the spacing between TE or TM modes with the same order number and with the differing mode number by 1 is approximated as $\Delta \nu \sim\left(c \tan ^{-1} \rho\right) /(2 \pi a \rho)$, where $\rho=\left(\eta^{2}-1\right)^{1 / 2}, a$ is radius, $\eta$ is refractive index of the sphere, and $c$ is the speed of light, ${ }^{9}$ which explains the experimental observation. In order to see the absolute mode numbers, we calculated the mode positions for the sphere of $d=8.1 \mu \mathrm{m}$ with $\eta=2.48$ and represented by a series of vertical lines in Fig. 2. In the notation, such as $\mathrm{TE}_{91,1}$, the first and second subscripts represent the mode $(l=91)$ and order $(n=1)$ numbers, respectively. The spectral peaks observed show good correlations with the predicted positions for the lower order modes.

While the cavity enhanced effects have widely been observed in optical experiments, such an effect has not been reported in cathode luminescence. Several differences could

Downloaded 10 Jul 2008 to 133.70 .80 .50 . Redistribution subject

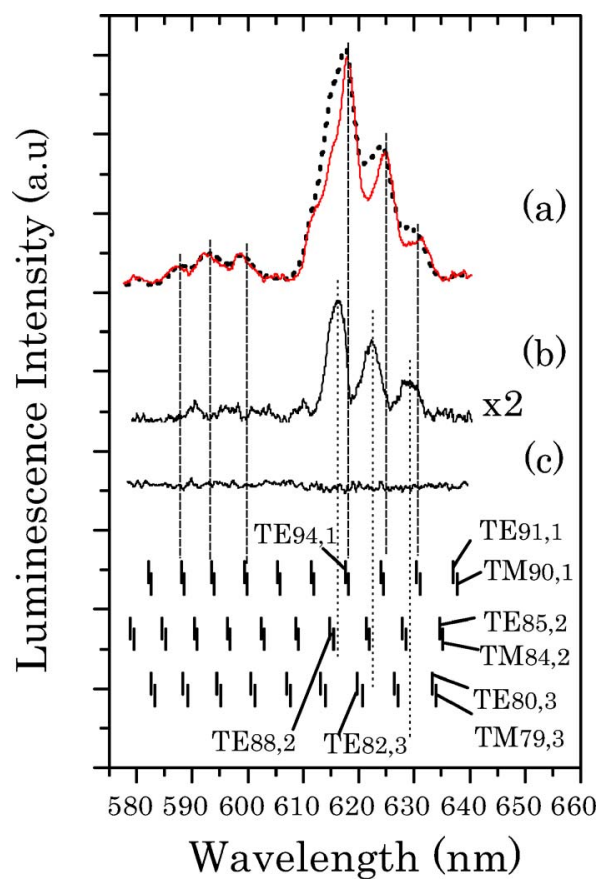

FIG. 2. Cathode luminescence spectra from $\mathrm{TiO}_{2}: \mathrm{Eu}^{3+}$ microsphere of $8.1 \mu \mathrm{m}$. (a) Solid and dotted lines are normalized spectra observed at $10 \mathrm{kV}$ (multiplied by 2.3) and $20 \mathrm{kV}$. Differential spectra: (b) $\Delta \mathrm{CL}=\mathrm{CL}(20 \mathrm{kV})$ $-2.3 \times \mathrm{CL}(10 \mathrm{kV})$ and $(\mathrm{c}) \Delta \mathrm{CL}=\mathrm{CL}(10 \mathrm{kV})-4.8 \times \mathrm{CL}(6 \mathrm{kV})$. A series of vertical lines (combs) represents the resonance wavelength of WGMs calculated for the microsphere of $d=8.1 \mu \mathrm{m}$ with refractive index of $\eta=2.48$. The order numbers are $n=1,2$, and 3 from top to bottom combs, while the mode number increases in a step of 1 from the long wavelength side to the short wavelength side.

appear between photoluminescence experiments and CL experiments. First, the CL experiment has the advantage that the electron beam can be focused below the optical wavelength and the local emission characteristics can be probed. ${ }^{16}$ Secondly, in the CL experiment, the depth dependent emission profiles can be examined through the control of the accelerating voltage. ${ }^{17}$ In order to demonstrate this advantage, we examined the dependence of CL spectra on the accelerating voltage. Figure 2(a) shows normalized spectra observed at 10 and $20 \mathrm{kV}$. We calculated the differential spectrum by subtracting the normalized spectrum of $10 \mathrm{kV}$ (multiplied by 2.3) from that of $20 \mathrm{kV}$ (multiplied by 1.0). It is seen that additional components appear when the voltage is increased up to $20 \mathrm{kV}$. Similar analysis was carried out for the spectra observed at $6 \mathrm{kV}$ (multiplied by 4.8 ) and $10 \mathrm{kV}$. In this case, the spectra at 6 and $10 \mathrm{kV}$ are essentially the same as shown in Fig. 2(c).

Figure 3 shows the luminescence intensity as a function of the accelerating voltage. From this figure, the dead voltage of luminescence quenching can be estimated as $5 \mathrm{kV}$. The corresponding penetration depth of electron beam through $\mathrm{TiO}_{2}$ at $5 \mathrm{kV}$ is estimated to be of the order of $0.1 \mu \mathrm{m}$ on the basis of the Feldman formalism, ${ }^{18}$ while this length increases up to $4 \mu \mathrm{m}$ at $20 \mathrm{kV}$. We can also calculate the spatial intensity distribution within the WGMs on the basis of Mie theorem. ${ }^{19}$ For WGMs around $620 \mathrm{~nm}$ with $n=1,2$, and 3 and $\mathrm{TE}_{94,1}, \mathrm{TE}_{88,2}$, and $\mathrm{TE}_{82,3}$ in Fig. 2, the radial positions of the intensity maximum from the sphere surface are calculated as $0.19,0.44$, and $0.65 \mu \mathrm{m}$, respectively. These values, together with the estimated penetration depth, suggest that at the accelerating voltage below $5 \mathrm{kV}$, the electron beam pen-
AlP license or copyright; see http://apl.aip.org/apl/copyright.jsp 


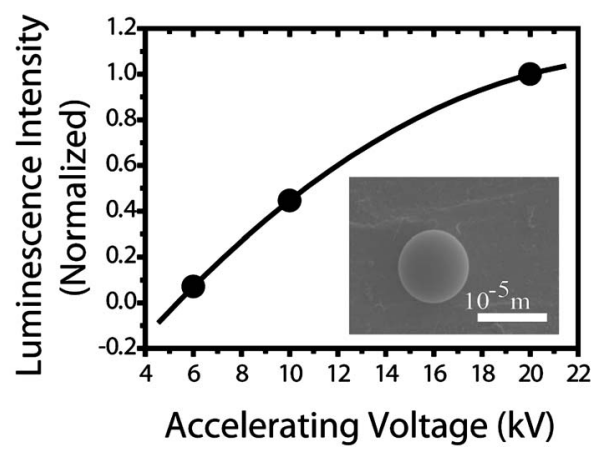

FIG. 3. Solid circles are normalized luminescence intensity in $\mathrm{TiO}_{2}: \mathrm{Eu}^{3+}$ microsphere of $8.1 \mu \mathrm{m}$ as a function of the accelerating voltage. The inset shows a scanning microscope photograph of a typical $\mathrm{TiO}_{2}: \mathrm{Eu}^{3+}$ microsphere.

etrates only the dead layer and does not excite the WGM efficiently, while at 6 or $10 \mathrm{kV}$, the beam excites the lower order number WGMs and shows the periodic structures in the CL spectra. When the accelerating voltage is further increased up to $20 \mathrm{kV}$, the beam excites the deep inner region of the sphere and additional components appear as shown in Fig. 2(b). The possible origin of the dead layer may be the surface defects or the charging up during the excitation of the $\mathrm{TiO}_{2}$ spheres.

In summary, we have observed WGM resonance modes in $\mathrm{CL}$ spectra in $\mathrm{TiO}_{2}: \mathrm{Eu}^{3+}$ microsphere. In the present experiment, we employed $\mathrm{TiO}_{2}$ microsphere, since the synthesis technique has well been developed. From the point of view of practical cathode phosphors, other host crystals such as $\mathrm{Y}_{2} \mathrm{O}_{3} \mathrm{~S}: \mathrm{Eu}^{3+}$ are interesting. Since the size of spheres synthesized by alkoxide method matches well with the typical size of fine display pixels, it is also interesting to design a microsphere laser array relevant to WGMs pumped by the field electron emitters. Such a coherent flat panel display could have potentiality in three dimensional displays or multidirectional displays.

The authors would like to thank Y. Nakanish, H. Kominami (Shizuoak University), and J. Seiyama (Bando Chemical Industries) for their fruitful discussions.

${ }^{1}$ Shigeo Shionoya, The Phosphor Handbook (CRC, Boca Raton, FL, 1999).

${ }^{2}$ M. Kottaisamy, K. Horikawa, H. Kominami, T. Aoki, N. Azuma, T. Nakamura, Y. Nakanishi, and Y. Hatanakab, J. Electrochem. Soc. 147, 1612 (2000).

${ }^{3}$ Jung-Chul Park, Hye-Kyung Moon, Dong-Kuk Kim, Song-Ho Byeon, and Bong-Chul Kim, Appl. Phys. Lett. 77, 2162 (2000).

${ }^{4}$ E. M. Purcell, Phys. Rev. 69, 681 (1946).

${ }^{5}$ A. J. Campillo, J. D. Eversole, and H.-B. Lin, Phys. Rev. Lett. 67, 437 (1991).

${ }^{6}$ K. J. Vahala, Nature (London) 424, 839 (2003).

${ }^{7}$ Peter Lodahl, A. Floris van Driel, Ivan S. Nikolaev, Arie Irman, Karin Overgaag, Daniël Vanmaekelbergh, and Willem L. Vos, Nature (London) 430, 645 (2004).

${ }^{8}$ C. F. Bohren and D. R. Huffman, Absorption and Scattering of Light by Small Particles (Wiley, New York, 1983).

${ }^{9}$ P. W. Barber and R. K. Chang, Optical Effects Associated With Small Particles (World Scientific, Singapore, 1988).

${ }^{10}$ Seongsik Chang, Nathan B. Rex, Richard K. Chang, Gabel Chong, and Louis J. Guido, Appl. Phys. Lett. 75, 166 (1999).

${ }^{11}$ L. H. Peng, C. Y. Lu, W. H. Wu, and S. L. Wang, Appl. Phys. Lett. 87, 161902 (2005)

${ }^{12}$ Silvija Gradečak, Fang Qian, Yat Li, Hong-Gyu Park, and Charles M. Lieber, Appl. Phys. Lett. 87, 173111 (2005).

${ }^{13}$ Xinhai Han, Guanzhong Wang, Qingtao Wang, Li Cao, Ruibin Liu, Bingsuo Zou, and J. G. Hou, Appl. Phys. Lett. 86, 223106 (2005).

${ }^{14}$ Anne Bagley Hardy, Wendell E. Rhine, and H. Kent Bowen, J. Am. Ceram. Soc. 76, 97 (1993).

${ }^{15} \mathrm{H}$. Ikari, K. Okanishi, M. Tomita, and T. Ishidate (unpublished).

${ }^{16}$ N. Yamamoto, H. Itoh, V. Grillo, S. F. Chichibu, S. Keller, J. S. Speck, S. P. DenBaars, U. K. Mishra, S. Nakamura, and G. Salviati, J. Appl. Phys. 94, 4315 (2003).

${ }^{17}$ H. C. Ong, A. S. K. Li, and G. T. Du, Appl. Phys. Lett. 78, 2667 (2001).

${ }^{18}$ Charles Feldman, Phys. Rev. 117, 455 (1960).

${ }^{19}$ Peter R. Conwell, Peter W. Barber, and Craig K. Rushforth, J. Opt. Soc. Am. A 1, 62 (1984). 